\title{
MOLECULAR TYPING FOR PUBLIC HEALTH PURPOSES
}

\author{
A Ammon (andrea.ammon@ecdc.europa.eu)1 \\ 1. European Centre for Disease Prevention and Control, Stockholm, Sweden
}

In this issue, seven networks/projects are presented that are dedicated to the molecular typing of bacteria (SeqNet: Staphylococcus aureus; MLVA-Net: Salmonella Typhimurium, Enterobacter sakazakii, Listeria monocytogenes; HARMONY: Staphylococcus aureus; DIPNET: Corynebacterium diphtheriae) or viruses (HepSEQ: hepatitis B virus; FBVE: noroviruses and other gastrointestinal viruses; MeaNS: measles). They represent only a few of an increasing number of typing networks. However, they illustrate a couple of relevant issues that need to be considered before implementing these methods for different public health purposes.

By providing appropriate discriminatory analyses, molecular typing can foster rapid and - depending on the method - even real-time early detection of dispersed international clusters/ outbreaks, the detection and investigation of transmission chains, the relatedness of strains, and the emergence of antimicrobial resistance and new evolving pathogenic strains. Molecular typing of infectious diseases, if routinely applied, can also complement traditional epidemiological surveillance. Moreover, analysis of molecular typing data can aid in studying the characteristics of a particular pathogen and its behaviour in a community of hosts, such as its spread over time and space, disease transmission dynamics, virulence factors influencing recurrence of infections, mutations and antigenic drifts of strains over time, and the development of drug resistance across strain generations.

Whereas the application of molecular typing during outbreaks and for the investigation of transmission chains is widely accepted, the use of these methods for routine surveillance is more debated, although there are successful examples, such as PulseNet in the United States [1]. However, before transferring a typing method from a research setting into wider use, a number of criteria proposed for the evaluation and validation must be considered.

Among those criteria are typeability, discriminatory power, epidemiological concordance and reproducibility. These characterise the "technical" appropriateness of a method for the typing of a specific pathogen [2]. For the implementation of an appropriate method into practice, a number of aspects need to be taken into consideration: the flexibility (to be used for more than one species); the rapidity; accessibility and overall cost; the ease of use, which includes the workload but also the interpretation of results; the amenability to computerised analysis and incorporation in electronic databases, which is important to combine the typing data with other epidemiological information [2].
For the inclusion of molecular typing data into surveillance at EU level, a few additional considerations are required before a typing method could be suggested for routine application:

- Typing data should provide essential information to achieve the surveillance objectives for the specific disease.

- The typing method should provide real-time information.

- The specific typing method/pathogen combination needs to be agreed upon among the laboratory experts.

- The molecular typing method is standardised in terms of the typing protocol and the nomenclature (or this standardisation is feasible), which allows comparison of data across laboratories and countries.

- External Quality Control needs to be established and regularly carried out.

- All Member States should have access to the agreed molecular typing method/pathogen, either by building up the capacity in their own country or by getting support from those Member States who have already developed the capacity.

The full synergy of combining molecular typing data and routine surveillance information and interpreting them jointly can contribute to improving and better targeting existing infectious disease prevention and control measures, and thus presents a clear benefit for public health and public health policy. However, the introduction of any of these methods will require a careful discussion between all involved stakeholders, which the European Centre for Disease Prevention and Control will encourage and foster, according to its mandate.

\section{References}

1. Tauxe RV. Molecular subtyping and the transformation of public health. Foodborne Pathog Dis. 2006 Spring;3(1):4-8.

2. Van Belkum A, Tassios PT, Dijkshoorn L, Haeggman S, Cookson B, Fry NK, et al; European Society of Clinical Microbiology and Infectious Diseases (ESCMID) Study Group on Epidemiological Markers (ESGEM). Guidelines for the validation and application of typing methods for use in bacterial epidemiology. Clin Microbiol Infect. 2007;13 Suppl 3:1-46.

This article was published on 8 May 2008.

Citation style for this article: Ammon A. Molecular typing for public health purposes. Euro Surveill. 2008;13(19):pii=18864. Available online: http://www.eurosurveillance.org/ ViewArticle.aspx?ArticleId $=18864$ 
